\title{
Fluid Flow Estimation with Multiscale Ensemble Filters Based on Motion Measurements Under Location Uncertainty
}

\author{
Sébastien Beyou ${ }^{1}$, Thomas Corpetti ${ }^{2}$, Sai Gorthi ${ }^{3}$ and Etienne Mémin ${ }^{1, *}$ \\ ${ }^{1}$ INRIA/FLUMINANCE, 35042 Rennes Cedex, France. \\ ${ }^{2}$ CNRS/LETG-Rennes-Costel, Campus Villejean Place du recteur Henri Le Moal \\ CS 2430735043 Rennes cedex, France. \\ ${ }^{3}$ Indian Institute of Space Science and Technology, Valiamala P.O., \\ Thiruvananthapuram - 695 547, Kerala India.
}

Received 6 December 2011; Accepted (in revised version) 11 September 2012

Available online 11 January 2013

\begin{abstract}
This paper proposes a novel multi-scale fluid flow data assimilation approach, which integrates and complements the advantages of a Bayesian sequential assimilation technique, the Weighted Ensemble Kalman filter (WEnKF) [27]. The data assimilation proposed in this work incorporates measurement brought by an efficient multiscale stochastic formulation of the well-known Lucas-Kanade (LK) estimator. This estimator has the great advantage to provide uncertainties associated to the motion measurements at different scales. The proposed assimilation scheme benefits from this multiscale uncertainty information and enables to enforce a physically plausible dynamical consistency of the estimated motion fields along the image sequence. Experimental evaluations are presented on synthetic and real fluid flow sequences.
\end{abstract}

AMS subject classifications: 65M10, 78A48

Key words: Data assimilation, stochastic filter, particle filters, fluid motion estimation.

\section{Introduction}

The analysis of geophysical fluid flows is of the utmost importance in domains such as oceanography, hydrology or meteorology for applications of forecasting, studies on climate changes or for monitoring hazards or events. The forecasting of such flows requires the precise knowledge of an initial condition which may be only accessible through the measurements of the system's state variables such as pressure, temperature, or fluid flow velocity. These data may be provided through dedicated probes or Lagrangian drifters

${ }^{*}$ Corresponding author. Email addresses: Sebastien.Beyou@inria.fr (S. Beyou), thomas . corpetti@univ-rennes2.fr (T. Corpetti), gorthisubrahmanyam@iist .ac.in (S. Gorthi), etienne.memin@inria.fr (E. Mémin) 
launch in the ocean or in the atmosphere. However, the coverage of such measurements is usually irregular and sometimes very sparse in underdeveloped regions or across oceans. At the opposite orbital or geostationary satellites provide a huge amount of image data, with a still increasing spatial and temporal resolution. Compared to in situ measurements (i.e., measure with local probes located inside the flow), satellite images provide a much more denser observation field. However they unfortunately offer only an indirect access to the physical quantities of interest and give rise consequently to difficult inverse problems to estimate characteristic features of the flow such as velocity fields or vorticity maps.

These kinematical information can be estimated from image sequences through motion estimation techniques. Motion estimation is an old problem in computer vision and a huge number of techniques has been proposed to estimate the motion on the image plane of a 3D scene. The best state-of-the-art approaches perform efficiently for the recovery of rigid scene motions $[28,31]$. They are generally built on strong photometric and motion hypothesis which prevent them to be sufficiently accurate for deformation metrology. Several motion estimators dedicated to the measurement of specific phenomenon such as fluid flows have been proposed in the literature (see [16] for a detailed overview). These estimators differ mainly on the smoothness function they are handling: first order penalization [29], second order div-curl regularization $[6,33]$, data-dependent $[3,7,24]$ or power law auto-similarity principles $[14,15]$. These methods provide accurate instantaneous displacements as they generally implement additional constraints imposed by the physics, as in $[6,11]$ and most of them are embedded into a multiscale formalism that enables capturing efficiently the large scales deformations [16]. However, those techniques generally still exhibit difficulties for mid to small scales measurements. Such artifacts may reveal particularly pronounced in regions with poor photometric contrasts where the smoothing prior takes the lead in the solution elaboration. In those regions the data come into play only at the boundary. Bad estimations or instabilities on the boundary vicinity are immediately echoed inside such regions. For large regions this may reveal problematic and constitutes a potential factor of instabilities along time of the estimates.

Dynamical consistency of the velocity measurements can be enforced by embedding the estimation problem within an image based assimilation process. Variational assimilations of image information have been recently considered for the estimation of fluid motion fields $[5,26]$. Those optimal control methods, though efficient, constitute batch methods, which requires forward and backward integrations of the dynamical system and the adjoint of the tangent linear dynamics respectively. The latter relies implicitly on a linearization of the dynamics and is adapted in practice for short time horizon. The constitution of this adjoint dynamics may turn out quite tedious in practice for complex dynamical models.

Stochastic filters are also well known alternative techniques for data assimilation. Opposite to the variational data assimilation framework, stochastic filtering has the great advantage to couple noisy data and a stochastic dynamics incorporating the unavoidable uncertainties we have on the system evolution. Such filters are also generally set up in a recursive way and through a Markovian property of the dynamics, they are at least theoretically less dependent on the initial condition. This relative independency with respect to the initial condition and their recursive structure along time are two important advan- 
tages. Obviously those filters require a precise modeling of the conditional distribution of the data given the true state, which is not always an easy task. Besides, the constitution of sound stochastic dynamics is in itself a difficult research problem for which no general theory exists. This filtering problem is in practice implemented either through particle filters or ensemble Kalman filters. The latter performs efficiently for linear systems but does not converge toward the true filtering distribution for nonlinear likelihood or dynamics [20]. As for the former, it has the advantage to deal with complex dynamics but is in its basic version limited to state spaces of moderate dimension [30], typically inferior to 100 and in any case three or four orders of magnitude lower than the dimension we are facing (corresponding to the image dimension e.g., $\sim\left[10^{5}, 10^{6}\right]$ ). Recently, a data assimilation procedure with the attempt of coupling the advantages of both filters has been proposed in embedding an Ensemble Kalman filter (EnKF) [27] into the particle filter (PF) framework. The approach is referred as Weighted Ensemble Kalman filter (WEnKF) [27]. The objective of this work consists to specify such a procedure from local noisy velocity measurements and their uncertainties and to define a multiscale extension of the filter.

To that end, we organize the paper as follows: Section 2 introduces the stochastic formulation of a local motion estimator based on the Lucas and Kanade technique [22] and referred as SLK in the rest of the paper. This modified version supplies the motion measurement at specific scale/resolution, along with their corresponding uncertainties. In Section 3, we present the Weighted Ensemble Kalman Filter (WEnKF) based fluid flow assimilation, relying on Navier-Stokes state dynamics and a linear observation model that uses SLK observations and uncertainties. Our novel multi-level WEnKF scheme, which is based on the same dynamical model but assimilates the observations at different scales, is proposed in Section 4. Some experimental validation and comparisons with the state-ofart fluid flow estimators are brought out in Section 5 and finally, Section 6 concludes the paper with a discussion to future directions.

\section{Stochastic Lucas-Kanade estimator}

Many possibilities are available to derive motion estimates from image data. This task, commonly named "optical flow", has been widely studied over the past three decades and huge amount of techniques are available. The reader can refer to [16] for a recent review on estimators dedicated to fluid flows and more generally to $[1,2,12]$ for presentation and comparative performance evaluations of some state-of-the-art techniques in computer vision. Interested readers may also refer to $[3,7,24]$, which provide recent trends for data based adaptive deformation estimation schemes.

Apart from some very specific applications where the observation process can be modeled in a precise way, almost all existing approaches are based on the well-known "opticalflow constraint equation" (OFCE), assuming a global conservation of the image luminance:

$$
\frac{d f(\underline{\mathbf{x}}, t)}{d t}=\frac{\partial f(\underline{\mathbf{x}}, t)}{\partial t}+\mathbf{v}(\underline{\mathbf{x}}, t) \cdot \nabla f(\underline{\mathbf{x}}, t)=0,
$$

where $\underline{\mathbf{x}}=(x, y)$ corresponds to the spatial coordinates, $\nabla$ is the gradient operator in the $x$ and $y$ directions and $f$ denotes the luminance function. 


\subsection{Lucas-Kanade estimator}

From relation (2.1), the seminal works of Horn and Schunck [17] and Lucas and Kanade [22] have been proposed. In the former method, the solution is obtained by minimizing a global energy functional. This functional combines the OFCE with a first-order spatial regularizer that promotes smooth motion fields over the whole image domain. The Lucas and Kanade technique is at the opposite a local approach that assumes a locally constant velocity on neighborhoods centered at each location $\underline{\mathbf{x}}$ of the image domain. At each point, the velocity is estimated through a local least squares formulation:

$$
\mathscr{I}(\underline{\mathbf{x}})=\left(\frac{\partial f(\underline{\mathbf{x}}, t)}{\partial t}+\mathbf{v}(\underline{\mathbf{x}}, t) \cdot \nabla f(\underline{\mathbf{x}}, t)\right)^{2}, \quad \mathbf{v}=\min _{\mathbf{v}=(u, v)^{T}} \int_{\Omega}\left[g_{\lambda}(\cdot) * \mathscr{I}(\cdot)\right](\underline{\mathbf{x}}) d \underline{\mathbf{x}},
$$

where $\Omega$ is the image domain, $g_{\lambda}$ is an isotropic Gaussian windowing function of standard deviation $\lambda$ in which the velocity $\mathbf{v}$ is assumed to be homogeneous. Solving the EulerLagrange equations associated to cost function (2.2), one gets (see Appendix 6 for details):

$$
\mathbf{v}=-\left(g_{\lambda} *\left[\begin{array}{cc}
f_{x}^{2} & f_{x} f_{y} \\
f_{x} f_{y} & f_{y}^{2}
\end{array}\right]\right)^{-1} g_{\lambda} *\left[\begin{array}{l}
f_{x} f_{t} \\
f_{y} f_{t}
\end{array}\right],
$$

where $f_{\bullet}=\partial f / \partial \bullet$. To guarantee a good conditioning of the previous matrix to invert, the spatial gradients must not vanish or be identical. The gaussian smoothing of the OFCE aims in fact at alleviating such a bad conditioning by capturing the spatial information at a scale related to $\lambda$. It is important to outline that the estimated velocity is hence intrinsically related to this scale $\lambda$.

From Horn \& Schunck and Lucas \& Kanade estimators, a huge number of methods based on diverse variations of these approaches has been proposed in the literature. Among them, the techniques focused first on the design of new regularization terms (able for instance to deal with occlusions, discontinuities or relying on physical grounds [16]) and second on the application of advanced minimization strategies. Surprisingly, apart for some specific applications devoted to some particular types of imagery (fluid, biology, infrared imagery, tomography, IRM, $\cdots$ ), only very few authors have worked on a generic alternative to the classical brightness consistency assumption and this despite to the fact it plays a crucial role in the motion estimation process.

In this paper, instead of relying on the OFCE to derive local motion measurements, we propose to formulate the motion estimation upon a stochastic version of the image luminance variation and to embed it in a local least squares formulation similar to the Lucas \& Kanade technique. This leads naturally to a continuous multiresolution formulation and enables not only to extract the motion fields at different resolutions but supplies uncertainties of those estimates as well. This is presented in the next sections.

\subsection{Luminance variation with uncertainties}

We first write the image luminance as the function of a stochastic process related to the position of image points. If one assumes, as illustrated in Fig. 1, that the velocity $\mathbf{v}$ 


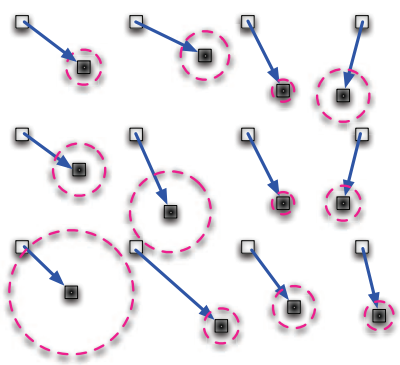

(a)

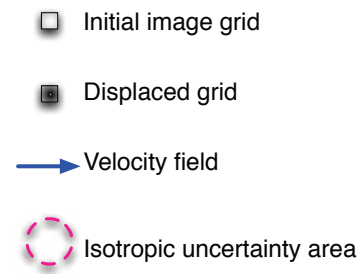

(b)

Figure 1: Displacement of the grid of points. The initial grid at time $t-1$ (white) is transported by the velocity field $\mathbf{v}$ to reach the configuration at time $t$ represented (black), up to some uncertainties (dashed lines).

to be estimated transports from time $t-1$ to time $t$ the grid of $2 \mathrm{D}$ points $\mathbf{X}_{t-1} \in \mathbb{R}^{2 m}$ to $\mathbf{X}_{t} \in \mathbb{R}^{2 m}$ up to an isotropic Brownian motion, we can write:

$$
\mathbf{X}_{t}=\mathbf{X}_{t-1}+\int_{t-1}^{t} \mathbf{v}(s) d s+\int_{t-1}^{t} \Sigma^{\frac{1}{2}}(s) d \mathbf{B}_{s}
$$

where $\mathbf{B}_{s}=\left(\mathbf{B}_{s}^{1}, \cdots, \mathbf{B}_{s}^{m}\right)^{T}$ is a multidimensional standard Brownian motion of $\mathbb{R}^{2 m}, \boldsymbol{\Sigma}$ a $(2 m \times 2 m)$ covariance matrix-valued function and $\mathbf{X}_{t}, \mathbf{X}_{t-1}$ represents the grid positions at time $t-1$ and $t$. Let us note the second right-hand integral has to be interpreted in a stochastic sense as Brownian motion is almost nowhere differentiable. In this work we shall rely on the Itô interpretation of such integral.

The luminance function $\mathbf{f}$ usually defined on spatial points $(x, y)$ at time $t$ is now defined on the grid as a map from $\mathbb{R}^{2 m} \times \mathbb{R}^{+}$into $\mathbb{R}^{m}$ and is assumed to be $C^{1,2}\left(\mathbb{R}^{2 m}, \mathbb{R}^{+}\right)$. Its differential is obtained following the differentiation rules of stochastic calculus (the so called Itô formulae) that gives the expression of the differential of any continuous deterministic function of an Itô diffusion of the form (2.4) (see [25] for an introduction to stochastic calculus):

$$
d \mathbf{f}\left(\mathbf{X}_{t}, t\right)=\frac{\partial \mathbf{f}}{\partial t} d t+\sum_{i=(1,2)} \frac{\partial \mathbf{f}\left(\mathbf{X}_{t}, t\right)}{\partial x_{i}} d X_{t}^{i}+\frac{1}{2} \sum_{(i, j) \in(1,2) \times(1,2)} \frac{\partial^{2} \mathbf{f}\left(\mathbf{X}_{t}, t\right)}{\partial x_{i} \partial x_{j}} d\left\langle X_{t}^{i}, X_{t}^{j}\right\rangle .
$$

The term $\left\langle X_{t}^{i}, X_{t}^{j}\right\rangle$ denotes the joint quadratic variations of $X^{i}$ and $X^{j}$ defined as the limit in probability over a partition $\left\{t_{1}, \cdots, t_{n}\right\}$ of $[0, t]$ with $t_{1}<t_{2}<\cdots<t_{n}$ and where denoting $\delta t_{i}=t_{i}-t_{i-1}$, the partition spacing defined as $\left|\delta_{n}\right|=\max _{i} \delta t_{i}$ is such that $\left|\delta_{n}\right| \rightarrow 0$ when $n \rightarrow \infty$ :

$$
\left\langle X_{t}^{i}, X_{t}^{j}\right\rangle=\lim _{\left|\delta_{n}\right| \rightarrow 0}^{\mathbb{P}} \sum_{t_{k} \leq t}\left(X_{t_{k-1}}^{i}-X_{t_{k}}^{i}\right)\left(X_{t_{k-1}}^{j}-X_{t_{k}}^{j}\right), \text { for } n \rightarrow \infty
$$

For Brownian motion, this quantity can be evaluated through the expectation of the righthand sum, an almost sure convergence is then obtained from the strong law of large num- 
bers (which implies convergence in probability). We have then the following identities:

$$
\begin{aligned}
& \left\langle B^{i}, B^{j}\right\rangle=\delta_{i j} t \text { a.s., } \\
& \langle h(t), h(t)\rangle=\left\langle h(t), d B^{i}\right\rangle=\left\langle B^{j}, h(t)\right\rangle=0 \text { a.s., }
\end{aligned}
$$

where $\delta_{i j}$ is the Kronecker symbol $\left(\delta_{i j}=1\right.$ if $i=j, \delta_{i j}=0$ otherwise $)$ and $h(t)$ is a deterministic function. Compared to classical differential calculus, new terms related to the Brownian random terms have been introduced in this stochastic formulation. In this work the stochastic part of (2.4) is defined as an isotropic uncertainty variance map $\sigma\left(\mathbf{X}_{t}, t\right): \mathbb{R}^{2 m} \times \mathbb{R}^{+} \rightarrow \mathbb{R}^{m}$

$$
\Sigma^{\frac{1}{2}}\left(\mathbf{X}_{t}, t\right) d \mathbf{B}_{t}=\operatorname{diag}\left(\sqrt{\sigma}\left(\mathbf{X}_{t}, t\right)\right) \otimes \mathbb{I}_{2} d \mathbf{B}_{t},
$$

where $\mathbb{I}_{2}$ is the $(2 \times 2)$ identity matrix and $\otimes$ denotes the Kronecker product. Applying Itô formula (2.5) to the isotropic uncertainty model yields a luminance variation defined as:

$$
\begin{aligned}
d \mathbf{f}\left(\mathbf{X}_{t}, t\right)=( & \left.\frac{\partial \mathbf{f}\left(\mathbf{X}_{t}, t\right)}{\partial t}+\nabla \mathbf{f}\left(\mathbf{X}_{t}, t\right) \cdot \mathbf{v}\left(\mathbf{X}_{t}, t\right)+\frac{1}{2} \sigma\left(\mathbf{X}_{t}, t\right) \Delta \mathbf{f}\left(\mathbf{X}_{t}, t\right)\right) d t \\
& +\sqrt{\sigma}\left(\mathbf{X}_{t}, t\right) \nabla \mathbf{f}\left(\mathbf{X}_{t}, t\right) \cdot d \mathbf{B}_{t} .
\end{aligned}
$$

It is straightforward to remark that the standard brightness consistency assumption is obtained from (2.8) using zero uncertainties $(\sigma=0)$. This stochastic formulation enables thus to introduce a softer constraint that takes into account an inherent measurement uncertainty due to the discrete grid representation for instance. In this simple model the set of grid points locations at time $t$ are thus conditionally independent with respect to their position at time $t-1$.

From relation (2.8), the conservation of the image luminance can be quite naturally expressed from the conditional expectation $\mathbb{E}\left(d \mathbf{f}\left(\mathbf{X}_{t}, t\right) \mid \mathbf{X}_{t-1}\right)$ between $t-1$ and $t$. To compute this term, we exploit the fact (as shown in Appendix 6) that the expectation of any function $\Psi\left(\mathbf{X}_{t}, t\right)$ of a stochastic process $d \mathbf{X}_{t}$ (as in (2.4)) knowing the grid $\mathbf{X}_{t-1}$ reads:

$$
\mathbb{E}\left(\Psi\left(\mathbf{X}_{t}, t\right) \mid \mathbf{X}_{t-1}\right)=\left(\widetilde{\Psi}(\cdot, t) * g_{\sigma}(\cdot)\right)\left(\mathbf{X}_{t-1}\right),
$$

where $g_{\sigma}\left(\mathbf{X}_{t}\right) \sim \mathscr{N}\left(\mathbf{X}_{t}, \boldsymbol{\Sigma}\right)$ is a multidimensional Gaussian centered in $\mathbf{X}_{t}$ with covariance $\boldsymbol{\Sigma}\left(\mathbf{X}_{t}, t\right)^{\dagger}$ and $\widetilde{\Psi}\left(\mathbf{X}_{t}, t\right)=\Psi\left(\mathbf{X}_{t}+\mathbf{v}, t\right)$. Relation (2.9) indicates that the expectation of a function $\Psi\left(\mathbf{X}_{t}, t\right)$ knowing the location $\mathbf{X}_{t-1}$ up to a Brownian uncertainty of variance $\sigma$ is obtained by a convolution of $\Psi\left(\mathbf{X}_{t-1}+\mathbf{v}, t\right)$ with a Gaussian kernel of mean $\mathbf{X}_{t}$ and covariance $\boldsymbol{\Sigma}$.

Assuming $\sigma$ known, our new luminance variation model $\mathbb{E}\left(d \mathbf{f}\left(\mathbf{X}_{t}, t\right) \mid \mathbf{X}_{t-1}\right)$ is hence defined as (removing the indexes $\left(\mathbf{X}_{t}, t\right)$ for sake of clarity):

$$
\mathbb{E}\left(d \mathbf{f}\left(\mathbf{X}_{t}, t\right) \mid \mathbf{X}_{t-1}\right)=g_{\sigma} *\left(\nabla \mathbf{f} \cdot \mathbf{v}+\frac{\partial \mathbf{f}}{\partial t}+\frac{1}{2} \sigma \Delta \mathbf{f}\right) .
$$

\footnotetext{
'Let us note that as the covariance $\boldsymbol{\Sigma}$ depends on the scalar $\sigma\left(\mathbf{X}_{t}, t\right)$, we note the Gaussian function as $g_{\sigma}$ to lighten the notation.
} 
Instead of using the OFCE as the basis constraint in the motion estimation process, we prefer to build our least squares estimator upon relation (2.10), which constitutes an OFCE model under location uncertainty. In the next section, we present a way to estimate such uncertainties $\sigma\left(\mathbf{X}_{t}, t\right)$.

\subsection{Uncertainty estimation}

Assuming an observed motion field $\mathbf{v}_{\mathbf{o b s}}$ that transports the luminance is accessible (we will describe in the next section an incremental framework involving such a motion), it is possible to estimate the uncertainty $\sigma\left(\mathbf{X}_{t}, t\right)$ by computing the quadratic variation of the luminance function $d \mathbf{f}$ between $t-1$ and $t$. Using the identities in (2.6), this yields:

$$
\left.d\left\langle\mathbf{f}\left(\mathbf{X}_{t}, t\right), \mathbf{f}\left(\mathbf{X}_{t}, t\right)\right\rangle=\sigma\left(\mathbf{X}_{t}, t\right) \| \nabla \mathbf{f}\left(\mathbf{X}_{t}, t\right)\right) \|^{2} .
$$

This quadratic variation can also be approximated from the luminance $\mathbf{f}$ by:

$$
d\left\langle\mathbf{f}\left(\mathbf{X}_{t}, t\right), \mathbf{f}\left(\mathbf{X}_{t}, t\right)\right\rangle \approx\left(\mathbf{f}\left(\mathbf{X}_{t}, t\right)-\mathbf{f}\left(\mathbf{X}_{t-1}, t-1\right)\right)^{2} .
$$

As convergence in probability implies convergence in distribution, the conditional expectation of both previous terms should be identical and one can estimate the variance by:

$$
\sigma\left(\mathbf{X}_{t}, t\right)=\frac{\mathbb{E}\left(\left(\mathbf{f}\left(\mathbf{X}_{t}, t\right)-\mathbf{f}\left(\mathbf{X}_{t-1}, t-1\right)\right)^{2}\right)}{\left.\mathbb{E}\left(\| \nabla \mathbf{f}\left(\mathbf{X}_{t}, t\right)\right) \|^{2}\right)}
$$

The expectation in the numerator and denominator are then computed at the displaced point $\mathbf{X}_{t-1}+\mathbf{v}_{\mathbf{o b s}}\left(\mathbf{X}_{t-1}\right)$ through the convolution of variance $\boldsymbol{\Sigma}\left(\mathbf{X}_{t-1}, t-1\right)$. A recursive estimation process is thus emerging from Eq. (2.13). Before entering into the details of the complete framework in Section 2.6, let us now rewrite the Lucas-Kanade estimation associated to our OFCE model under location uncertainty (2.10).

\subsection{Stochastic Lucas-Kanade estimator}

A local motion estimator relying on the new observation model defined in (2.10) can be now expressed as the following minimization problem (dropping the coordinates $\left(\mathbf{X}_{t}, t\right)$ ):

$$
\min _{\mathbf{v}} g_{\lambda} * g_{\sigma} *\left(\nabla \mathbf{f} \cdot \mathbf{v}+\frac{\partial \mathbf{f}}{\partial t}+\frac{1}{2} \sigma \Delta \mathbf{f}\right)^{2} .
$$

In a similar way to the standard Lucas and Kanade estimator (see Appendix 6) this yields the following relation, for the velocity estimate of a given grid point:

$$
\left(g_{\lambda} * g_{\sigma}\left[\begin{array}{cc}
\mathbf{f}_{x}^{2} & \mathbf{f}_{x} \mathbf{f}_{y} \\
\mathbf{f}_{x} \mathbf{f}_{y} & \mathbf{f}_{y}^{2}
\end{array}\right]\right) \mathbf{v}=-g_{\lambda} * g_{\sigma} *\left(\frac{1}{2} \sigma \Delta \mathbf{f}+\mathbf{f}_{t}\right)\left[\begin{array}{c}
\mathbf{f}_{x} \\
\mathbf{f}_{y}
\end{array}\right] .
$$

In this model the Gaussian windowing function $g_{\lambda}$ of mean $\mathbf{X}_{t}$ and covariance $\lambda I_{2 m}$ can be interpreted as the distribution of a new isotropic constant uncertainty term related to the grid resolution and independent of the motion uncertainties depending on the image data. 
Let us note otherwise that the data model (2.14) introduces a Laplacian diffusion with a minus sign (antidiffusion effect). This numerically unstable effect is however counterbalanced by the Gaussian smoothing $g_{\sigma}$ with a variance that is twice the antidiffusion coefficient. The supplementary filtering due to the Gaussian windowing function $g_{\lambda}$ introduced to counter the illposed nature of the brightness consistency assumption, further stabilizes the process. In practice, the choice of the convolution kernel standard deviation $\lambda$ is crucial: a large value of $\lambda$ will remove all details whereas a small value is likely to be unstable and may lead in an extreme case to an illposed problem. In addition, such motion estimation procedure, based on a linearized version of the displaced frame difference, leads to inaccurate measurements of large displacements when the linear assumption of the brightness consistency breaks (high photometric gradients and/or large displacements). To prevent such limitations many authors have proposed to embed such estimation procedures within a pyramidal setup. However, a pyramidal representation requires Gaussian filtering, sampling and interpolation of the input data which introduce potential artifacts that may spoil the estimation. Interpreting the windowing function convolution as associated to the computation of the expectation of a grid uncertainty random term will allow us defining an original continuous multiresolution framework. This is presented in the next paragraph.

\subsection{Multiresolution version}

A multiresolution scheme consists in redefining the problem on a grid $\mathbf{X}^{\ell}$ which can be viewed as a coarse representation of the initial grid $\mathbf{X}^{0}=\mathbf{X}$ with a Brownian isotropic uncertainty of constant variance $\lambda^{\ell}$ : from the initial pixel grid $\mathbf{X}^{0}=\mathbf{X}$, which corresponds to the whole image domain, the problem is redefined on a grid $\mathbf{X}^{\ell}$ associated to a coarse representation of $\mathbf{X}$. This reads

$$
\mathbf{X}^{\ell}=\mathbf{X}^{0}+\sqrt{\lambda}^{\ell} \mathbb{I}_{2} d \mathbf{B}_{t} .
$$

The motion $\mathbf{v}\left(\mathbf{X}_{t-1}^{\ell}, t-1\right)$ on this grid should minimize the expectation $\mathbb{E}\left(d \mathbf{f}^{2}\left(\mathbf{X}_{t}^{\ell}, t\right) \mid \mathbf{X}^{0}\right)$ which is equivalent (see Appendix 6) to a convolution of $d \mathbf{f}^{2}(\cdot, t)$ with the isotropic gaussian $\mathscr{N}\left(0, \lambda^{\ell}\right)$. Therefore, one gets exactly the system in (2.14) which is solved locally by inverting the system in (2.15). The main advantage of such a formulation of the multiresolution setup is to naturally get rid of the use of a pyramidal image representation. Instead of dealing with successive decimations of factor 2 of the initial image to fix the different multiresolution levels, the evolutions of the levels $\ell$ are much flexible here. This framework may be interpreted as a scale space representation of the extended brightness consistency assumption [21] stemming in our case rigorously from an uncertainty analysis of the point location. Let us note the heat equation, which correspond to the backward Kolmogorov equations associated to (2.16), constitutes formally the connection point between the traditional scale space representation and this multiresolution setup.

From the Stochastic Lucas-Kanade normal equations (2.15), the estimation of uncertainties from $\sigma$ of relation (2.13) and the above multiresolution framework, one can define a complete incremental framework presented in the next section. 


\subsection{Incremental framework}

The local estimator proposed here comprises a specific multiresolution scheme where at each level, an incremental framework is defined to estimate as accurately as possible the uncertainties. The overall scheme is figured in Algorithm 2.1.

Algorithm 2.1: Stochastic Lucas-Kanade: incremental framework

1. Initializations:

- Fix an initial resolution level $\ell=L$

- Define $\tilde{\mathbf{f}}\left(\mathbf{X}_{t-1}, t\right):=\mathbf{f}\left(\mathbf{X}_{t-1}, t\right) ; \mathbf{v}=0$;

2. Estimation for the level $\ell$

(a) Initializations:

- $n=1 ; \mathbf{v}^{0}=0$;

- Fix an initial $\sigma^{0}$

(b) Estimate $\sigma^{n}$ by relation (2.13)

(c) Find $\mathbf{v}^{n}$ by local inversions of the system (2.15)

(d) Update motion field: $\mathbf{v}:=\mathbf{v}+\mathbf{v}^{n}$

(e) Warp the image $\mathbf{f}\left(\mathbf{X}_{t}, t\right): \tilde{\mathbf{f}}\left(\mathbf{X}_{t-1}\right)=\mathbf{f}\left(\mathbf{X}_{t-1}+\mathbf{v}, t\right)$

(f) $n:=n+1$

(g) Loop to step (b) until convergence $\left(\left|\mathbf{v}^{n}\right|<\epsilon\right)$;

3. Decrease the multiresolution level: $\lambda^{\ell}=\alpha \lambda^{\ell+1}$ where $\alpha<1$ is fixed by the user;

4. Loop to Step 2 until convergence $\left(\lambda^{\ell}<\lambda_{\text {min }}^{\ell}\right)$.

This framework is a natural and simple implementation of a local motion estimation technique using the proposed isotropic uncertainty model for the evolution of the luminance. The values $\sigma$ provide us a spatial distribution of the motion estimate uncertainties. These uncertainties constitute a very useful piece of information when coping with the filtering of noisy motion measurements. Let us now turn to the presentation of the stochastic filter considered in this work.

\section{Monte Carlo implementation of stochastic filtering with the Weighted-Ensemble Kalman filter}

In this section we briefly review the main principles driving the construction of the Weighted Ensemble Kalman filter, proposed in [27] and discuss its advantages and limitations in the context of fluid flow analysis. This technique is a particle implementation of a nonlinear stochastic filtering problem build upon an ensemble Kalman update stage. Let us first introduce basic elements of stochastic filtering. 


\subsection{Stochastic filtering, filtering distribution}

Stochastic filters aim at estimating the posterior probability distribution $p\left(\mathbf{x}_{0: k} \mid \mathbf{y}_{1: k}\right)$ of a state variable trajectory $\mathbf{x}_{0: k}$ starting from an initial state $\mathbf{x}_{0} \in \mathbb{R}^{n}$ up to the current time $k$ given a complete measurements trajectory $\mathbf{y}_{1: k}$. The state variable trajectory is obtained through the integration of a dynamical system:

$$
\mathbf{x}_{t}=\mathbf{M}\left(\mathbf{x}_{t-\delta t}\right)+\boldsymbol{\eta}_{t}
$$

where $\mathbf{M}$ denotes a deterministic linear/nonlinear dynamical operator, corresponding to a discrete representation (through numerical integration with time step $\delta t$ ) of a physical conservation law describing the state evolution. Parameter $\boldsymbol{\eta}_{t}$ is usually a white Gaussian noise of covariance $\mathbf{Q}_{\delta t}$, that accounts for the uncertainties in the deterministic state model. However, as the true initial state is unknown, observation $\mathbf{y}_{k} \in \mathbb{R}^{m}$ of the state occurring at discrete instants are assumed to be available. These observations and the state variable are linked through a measurement equation:

$$
\mathbf{y}_{k}=\mathbf{H}\left(\mathbf{x}_{k}\right)+\boldsymbol{r}_{k},
$$

where $\gamma_{k}$, the observation noise, is a white Gaussian noise with covariance matrix $\mathbf{R}$ and operator $\mathbf{H}$ stands for the linear/nonlinear mapping from the state variable space to the observation space. We note that the (integration) time step used for the state variable dynamics $\delta t$ is usually much smaller (about 10-100 times), than the latency $\delta k$ between two subsequent measurements. A sequence of measurements or observations from time 1 to $k$ will be denoted by a set of vectors of dimension $m$ as: $\mathbf{y}_{1: k}=\left\{\mathbf{y}_{i}, i=1, \cdots, k\right\}$ where the latency between two successive measurements is arbitrarily set to $\delta k=1$.

A recursive expression of the filtering distribution $p\left(\mathbf{x}_{0: k} \mid \mathbf{y}_{1: k}\right)$, describing the distribution of the unknown hidden Markov process conditioned upon the whole set of past observations $\mathbf{y}_{1: k}$, can be obtained from Bayes' law and the assumption that the measurements depend only on the current state (e.g., $p\left(\mathbf{y}_{k} \mid \mathbf{x}_{0: k}, \mathbf{y}_{1: k-1}\right)=p\left(\mathbf{y}_{k} \mid \mathbf{x}_{k}\right)$ ):

$$
\begin{aligned}
p\left(\mathbf{x}_{0: k} \mid \mathbf{y}_{1: k}\right) p\left(\mathbf{y}_{k} \mid \mathbf{y}_{1: k-1}\right) & =p\left(\mathbf{x}_{0: k}, \mathbf{y}_{k} \mid \mathbf{y}_{1: k-1}\right) \\
& =p\left(\mathbf{y}_{k} \mid \mathbf{x}_{0: k}, \mathbf{y}_{1: k-1}\right) p\left(\mathbf{x}_{0: k} \mid \mathbf{y}_{1: k-1}\right) \\
& =p\left(\mathbf{y}_{k} \mid \mathbf{x}_{k}\right) p\left(\mathbf{x}_{k} \mid \mathbf{x}_{0: k-1}, \mathbf{y}_{1: k-1}\right) p\left(\mathbf{x}_{0: k-1} \mid \mathbf{y}_{1: k-1}\right)
\end{aligned}
$$

From Markoviannity of the dynamic system, we finally get the sought recursive expression:

$$
p\left(\mathbf{x}_{0: k} \mid \mathbf{y}_{1: k}\right)=p\left(\mathbf{x}_{0: k-1} \mid \mathbf{y}_{1: k-1}\right) \frac{p\left(\mathbf{y}_{k} \mid \mathbf{x}_{k}\right) p\left(\mathbf{x}_{k} \mid \mathbf{x}_{k-1}\right)}{p\left(\mathbf{y}_{k} \mid \mathbf{y}_{1: k-1}\right)}
$$

For linear models, this recursive equation can be solved through the Kalman filter. This is described in the next section. 


\subsection{Linear Gaussian models and the Kalman filter}

For a Gaussian initial distribution, additive Gaussian noises, linear dynamics and linear measurement operator, denoted by $\mathbf{M}$ and $\mathbf{H}$ respectively, the distribution $p\left(\mathbf{x}_{k} \mid \mathbf{y}_{1: k}\right)$ remains a Gaussian distribution whose first and second moment, $\mathbf{x}_{k}^{a}=\mathbb{E}\left(\mathbf{x}_{k} \mid \mathbf{y}_{1: k}\right)$ and $\mathbf{P}_{k}^{a}=\mathbb{E}\left(\left(\mathbf{x}-\mathbf{x}_{k}^{a}\right)\left(\mathbf{x}-\mathbf{x}_{k}^{a}\right)^{T} \mid \mathbf{y}_{1: k}\right)$, can be explicitly computed from the well known recursive Kalman equations [18]:

$$
\mathbf{x}_{k}^{f}=\mathbf{M} \mathbf{x}_{k-1}^{a}, \quad \mathbf{P}_{k}^{f}=\mathbf{M P}_{k-1}^{a} \mathbf{M}^{T}+\mathbf{Q}_{k},
$$

and

$$
\begin{aligned}
\mathbf{K}_{k} & =\mathbf{P}_{k}^{f} \mathbf{H}^{T}\left(\mathbf{H P}_{k}^{f} \mathbf{H}^{T}+\mathbf{R}\right)^{-1}, \\
\mathbf{x}_{k}^{a} & =\mathbf{x}_{k}^{f}+\mathbf{K}_{k}\left(\mathbf{y}_{k}-\mathbf{H} \mathbf{x}_{k}^{f}\right), \\
\mathbf{P}_{k}^{a} & =\left(\mathbb{I}-\mathbf{K}_{k} \mathbf{H}\right) \mathbf{P}_{k}^{f},
\end{aligned}
$$

here superscripts $f$ and $a$ on state variable expectation and covariance denote the respective quantities before and after analysis (update) at time $k$, respectively. The prediction or forecast step (3.4) brings forward the first two moments of the state vector, from its previous time step $k-1$, through the dynamical model parameters, while the analysis or the correction step (3.5c) provides the first two moments of the state characterizing the Gaussian filtering distribution at time $k$. The matrix $\mathbf{K}_{k}$ is referred to as the Kalman gain matrix and defines the weighting between the forecast state and the innovation brought by the observation: $\mathbf{y}_{k}-\mathbf{H x}_{k}^{f}$.

In the case of nonlinear models, it is possible to approximate the posterior distribution with a set of particles. This is presented in the next section.

\subsection{Particle implementation of the nonlinear filtering}

For nonlinear dynamics or nonlinear measurement equation, a direct sampling from the filtering distribution is impossible since it would require the complete knowledge of the filtering distribution - which is in the general case a non Gaussian multimodal distribution - at a previous time.

Particle filtering techniques introduce a discrete approximation of the sought density as a sum of $N$ weighted Diracs:

$$
p\left(\mathbf{x}_{0: k} \mid \mathbf{y}_{1: k}\right) \approx \sum_{i=1}^{N} w_{k}^{(i)} \delta_{\mathbf{x}_{0: k}}\left(\mathbf{x}_{0: k}\right)
$$

centered on hypothesized locations of the state space sampled from a proposal distribution, $\pi$, (also called the importance distribution) approximating the true filtering distribution $p\left(\mathbf{x}_{0: k} \mid \mathbf{y}_{1: k}\right)$. Each sample is then weighted by $w_{k}^{(i)}$, accounting for the ratio between the two distributions. A great variety of importance functions can be chosen (with the only restriction that its support contains the filtering distribution one). Obviously the closer it 
is to the targeted filtering distribution the better the results. Under a weak hypotheses assuming a recursive factorization form of the importance function, the importance ratio can be recursively defined as:

$$
w_{k}^{(i)} \propto w_{k-1}^{(i)} \frac{p\left(\mathbf{y}_{k} \mid \mathbf{x}_{k}^{(i)}\right) p\left(\mathbf{x}_{k}^{(i)} \mid \mathbf{x}_{k-1}^{(i)}\right)}{\pi\left(\mathbf{x}_{k}^{(i)} \mid \mathbf{x}_{0: k-1}^{(i)}, \mathbf{y}_{1: k}\right)} .
$$

By propagating the particles from time $k-1$ through the proposal density $\pi\left(\mathbf{x}_{k}^{(i)} \mid \mathbf{x}_{0: k-1}^{(i)}, \mathbf{y}_{1: k}\right)$ and by weighting the sampled states with $w_{k}^{(i)}$, a sampling of the filtering law is obtained. When the proposal distribution $\pi$ is set to the prior (i.e., $\pi\left(\mathbf{x}_{k}^{(i)} \mid \mathbf{x}_{0: k-1}^{(i)}, \mathbf{y}_{1: k}\right)=p\left(\mathbf{x}_{k}^{(i)} \mid \mathbf{x}_{k-1}^{(i)}\right)$, the weights updating rule (3.7) simplifies to the data likelihood $p\left(\mathbf{y}_{k} \mid \mathbf{x}_{k}^{(i)}\right)$. This particular instance of the particle filter is called the Bootstrap filter or sequential importance resampling (SIR) filter [13]. Due to its simplicity it is the most commonly used particle filter. Nevertheless, such an importance function does not take into account the current observation and depends only weakly on the past data through the filtering distribution estimated at the previous instant. High dimensional probability distribution spaces being excruciatingly difficult to sample, it is very important to devise an importance function that enables focusing on the most meaningful areas of the state space. To that end it is essential to consider proposal distributions that take into account more significantly the past and current measurements. Along this idea, the weighted ensemble Kalman filter defines the proposal distribution from the sampling mechanisms of ensemble Kalman filtering techniques.

\subsection{Ensemble Kalman filtering}

The Ensemble Kalman filter [9] can be interpreted as a Monte Carlo implementation of the Kalman filter recursion for the propagation of the two first moments. The Ensemble filter relies hence intrinsically on a Gaussian approximation of the filtering distribution.

More precisely, let us assume that we have sampled $N$ members from the initial filtering distribution $p\left(\mathbf{x}_{0} \mid \mathbf{y}_{0}\right)$, denoted by $\mathbf{x}_{0}^{(i)}, i=1, \cdots, N$. Propagating these samples, iteratively, through the Kalman prediction and correction steps, provides us the Gaussian approximations of the prediction and filtering distributions.

The prediction step consists in propagating the ensemble members $\mathbf{x}_{k-1}^{a,(i)}$ and their associated uncertainties (noise) through the state dynamics in order to obtain a predicted particles or forecast ensemble as:

$$
\mathbf{x}_{k}^{f,(i)}=\sum_{t=k-1}^{k-\delta t}\left(\mathbf{M}\left(\mathbf{x}_{t}^{f,(i)}\right)+\eta_{t+\delta t}^{(i)}\right), \quad \mathbf{x}_{k-1}^{f,(i)}=\mathbf{x}_{k-1}^{a,(i)} .
$$

From this, the empirical mean, $\overline{\mathbf{x}}_{k}^{f}$, of the forecast ensemble and the corresponding empirical forecast covariance matrix $\mathbf{P}_{k}^{f_{e}}$ are computed. Using this ensemble based forecast covariance, an ensemble based Kalman gain matrix $\mathbf{K}_{k}^{e}$ can be computed. With this Kalman 
gain and the observation model the forecast ensemble members are then corrected towards the current observation.

This correction consists to update the forecast ensemble members $\mathbf{x}_{k}^{f,(i)}$, through the Kalman update Eqs. (3.4)-(3.5c), with a set of perturbed observation $\mathbf{y}_{k}+\boldsymbol{\gamma}_{k}^{(i)}$ obtained from samples of the observation noise $\left\{\gamma_{k}^{(i)}, i=1, \cdots, N\right\}$. This supplies an analysis ensemble members $\left\{\mathbf{x}_{k}^{a,(i)}, i=1, \cdots, N\right\}$ defined as:

$$
\mathbf{x}_{k}^{a,(i)}=\mathbf{x}_{k}^{f,(i)}+\mathbf{K}_{k}^{e}\left(\mathbf{y}_{k}+\boldsymbol{r}_{k}^{(i)}-\mathbf{H} \mathbf{x}_{k}^{f,(i)}\right) .
$$

Here, we note that, in the Kalman gain or in the update stage, computation of the high dimensional covariance matrix or pseudoinverse of the $n \times n$ covariance term, $\left(\mathbf{H P}_{k}^{f_{e}} \mathbf{H}^{T}+\right.$ $\mathbf{R})^{-1}$ are never explicitly computed nor stored. Rather, Kalman gain and update are efficiently implemented by defining and employing matrices with ensemble of perturbations. In most of the geophysical applications, the size $n$ of the state vector related usually to temperature, pressure or velocity fields is of much higher dimension than the number of samples $N$ used in EnKF. i.e., $n \gg N$. Thus, handling the perturbation matrices (instead of the actual corresponding covariance matrices) approximately brings down the number of operations from $\mathscr{O}\left(n^{2}\right)$ to $\mathscr{O}(n N)$. The inverse needed in the Kalman gain can be efficiently computed through the singular value decomposition of a $n \times N$ matrix [27].

\subsection{Weighted EnKF}

Starting from the descriptions of the previous section, a hybrid filtering procedure that takes advantage of both the particle filter and the EnKF can be devised. We briefly describe the approach proposed in [27].

The importance sampling principle indicates that a wide range of proposal distributions can be considered. We will experimentally show that a proposal distribution defined by the EnKF procedure constitutes an efficient proposal mechanism for particle filter techniques in high dimensional spaces.

Relying on the usual assumption of the EnKF (i.e., considering the dynamics as a discrete Gaussian system), the conditional distribution $p\left(\mathbf{x}_{k} \mid \mathbf{x}_{k-1}^{(i)}, \mathbf{y}_{k}\right)$ can be approached by a Gaussian distribution of respective mean and covariance [27]:

$$
\bar{\mu}_{k}^{(i)}=\left(\mathbb{I}-\mathbf{K}_{k}^{e} \mathbf{H}\right) \sum_{t=k-1}^{k-\Delta t} M\left(\mathbf{x}_{t}^{f,(i)}\right)+\mathbf{K}_{k}^{e} \mathbf{y}_{k}, \quad \Sigma_{k}^{e}=\left(\mathbb{I}-\mathbf{K}_{k}^{e} \mathbf{H}\right) \mathbf{P}_{k}^{f_{e}} .
$$

This distribution provides us a natural expression for the proposal distribution. In order to make the estimation of the filtering distribution exact (up to the sampling), each member of the ensemble must be weighted at each instant, $k$, with appropriate weights, $w_{k}^{(i)}$, defined from (3.7). For high dimensional systems represented on the basis of a very small number of particles, the weights simplify as [27]:

$$
w_{k}^{(i)} \propto p\left(\mathbf{y}_{k}^{o} \mid \mathbf{x}_{k}^{(i)}\right) \text { and } \sum_{i=1}^{N} w_{k}^{(i)}=1
$$


Therefore, the Weighted ensemble Kalman filter (WEnKF) procedure can be simply summarized by the Algorithm 3.1.

Algorithm 3.1: The WEnKF algorithm, one iteration

Require: Ensemble at instant $k-1:\left\{\mathbf{x}_{k-1}^{(i)}, i=1, \cdots, N\right\}$

observations $\mathbf{y}_{k}$

Ensure: Ensemble at time $k:\left\{\mathbf{x}_{k}^{(i)}, i=1, \cdots, N\right\}$

EnKF step: Get $\mathbf{x}_{k}^{(i)}$ from the assimilation of $\mathbf{y}_{k}^{o}$ with an EnKF procedure;

Compute the weights $w_{k}^{(i)}$ according to (3.11);

Resample: For $j=1, \cdots, N$, sample with replacement index $I(j)$ from discrete probability $\left\{w_{k}^{(i)}, i=1, \cdots, N\right\}$ over $\{1, \cdots, N\}$ and set $\mathbf{x}_{k}^{(j)}=\mathbf{x}_{k}^{I(j)}$.

The next section presents the way we combine SLK (Stochastic Lucas Kanade introduced in Section 2) observations with WEnKF to perform multiscale fluid motion estimation.

\section{Multiscale WEnKF assimilation of SLK observations}

In what follows, we first detail the dynamical model, the observation model and the strategy we adopt to incorporate the uncertainties supplied by the SLK estimator. In a second step, the Section 4.2 presents its multiscale extension to extract consistent fluid motion estimates.

\subsection{Models and uncertainties}

The objective being to estimate velocities related to $2 \mathrm{D}$ incompressible fluid flows, we prefer to depict the velocity $\mathbf{v}=[u, v]^{T}$ by the scalar $\xi=\partial v / \partial x-\partial u / \partial y=v_{x}-u_{y}$ that represents the vorticity. For such incompressible flows, the velocity field can easily be recovered from its vorticity using the Biot-Savart kernel: $\mathbf{v}=\nabla^{\perp} G * \xi$ where $G$ denotes the Green kernel $\left(G=\frac{1}{2 \pi} \ln (|x|)\right)$ associated to the Laplacian operator.

\subsubsection{Dynamical model}

As in this work we considered only 2D incompressible fluid flows, we will rely for the dynamics on the vorticity-velocity formulation of the Navier-Stokes equation with a stochastic forcing function:

$$
d \xi=-\nabla \xi \cdot \mathbf{v} d t+v \Delta \xi d t+\eta d B_{t},
$$

where $v$ is the kinematic viscosity and $\eta d B$ is a random forcing term (introduced later in this section). The numerical simulation of this dynamical model is detailed in [27]. We will note $\xi_{k} \in \mathbb{R}^{|\Omega|}$ the finite dimensional state vector describing at instant $k$ the vorticity map over the spatial domain $\Omega$ and $\mathbf{v}\left(\xi_{k}\right)$ will denote the corresponding velocity field (where 
an explicit dependance on the vorticity is emphasized to indicate the nonlinear nature of dynamics (4.1)).

\subsubsection{Observation model}

The measurements on which we will rely on are set directly as the curl map (i.e., vorticity) of the SLK velocity estimates (cf. Section 2). Assuming the observation is a corrupted version of the true vorticity map (state), we define the observation model as:

$$
\widetilde{\xi}_{k}=\xi_{k}+\gamma_{k},
$$

where $\gamma_{k}$ is a Gaussian random field whose variance is fixed to the spatially varying uncertainties associated to the measurements. The constitution of the random fields associated to the dynamics and measurement noises are detailed in the next sections.

\subsubsection{Noise and uncertainties}

To simulate the random forcing term $d \mathbf{B}$ in the dynamics (4.1) and the random field of the observation model (4.2), homogeneous Gaussian fields, correlated in space but uncorrelated in time, are used. Their covariance have a general form given by:

$$
\mathbf{Q}(\underline{\mathbf{r}}, \tau)=\mathbb{E}\left[d \mathbf{B}(\underline{\mathbf{x}}, t) d \mathbf{B}^{T}(\underline{\mathbf{x}}+\underline{\mathbf{r}}, t+\tau)\right]=\mathbf{g}_{\lambda}(\underline{\mathbf{r}}) d t \delta(\tau),
$$

where $\mathbf{g}_{\lambda}(\underline{\mathbf{r}})$ describes the spatial correlation structure with cutoff parameter $\lambda$ beyond which two points are uncorrelated and $\delta$ denotes the Dirac distribution (indicating the random fields are temporally uncorrelated). These random fields are in practice sampled in the Fourier domain or on wavelet basis $[8,23]$.

\subsubsection{Dynamics noise}

More precisely, for the noise associated to the dynamics, we consider a divergence free random field that mimics real turbulent flows. This is done by exhibiting within an inertial range of scales an energy spectrum defined as a power law model $\|\mathbf{x}\|^{\zeta / 2}$ with an exponent $0<\zeta<2$. The covariance tensor associated to this random field, usually referred in the literature as Kraichnan model [19], can be written more conveniently in terms of its spectral representation:

$$
\mathbb{E}\left(\eta d \boldsymbol{B}_{t}(\underline{\mathbf{x}}) \eta d \boldsymbol{B}_{s}(\underline{\mathbf{y}})^{T}\right)=\delta(t-s) \frac{d t}{(2 \pi)^{d}} \int_{\mathbb{R}^{d}} e^{i \mathbf{k}(\underline{\mathbf{x}}-\underline{\mathbf{y}})} \widehat{\mathbf{Q}}(\mathbf{k}) d \mathbf{k},
$$

where the spectral correlation tensor in dimension $d$ is defined as:

$$
\widehat{\mathbf{Q}}(\mathbf{k})_{i j}=C_{\zeta}^{2}|\mathbf{k}|^{-\zeta-d}\left(\delta_{i j}-\frac{k_{i} k_{j}}{|\mathbf{k}|^{2}}\right)\left(\widehat{\psi_{\gamma}^{\kappa}}\right)^{2} .
$$

In the previous relation, $C_{\zeta}$ corresponds to a dissipative constant fixed on dimensional considerations and function $\psi_{\kappa}^{\gamma}$ defines a band-pass cut-off function, allowing to keep only 
a given range of scales, corresponding to the so-called inertial scale range defined between the short dissipative scale $\ell_{D}=1 / \kappa$ and the large integral scale $L=1 / \gamma$ at which the forcing takes place. The variance of this homogeneous random field is

$$
\frac{C_{\zeta}^{2} d t}{(2 \pi)^{d}} \int_{\mathbb{R}^{d}}|\mathbf{k}|^{-\zeta-d}\left(\delta_{i j}-\frac{k_{i} k_{j}}{|\mathbf{k}|^{2}}\right)\left(\widehat{\psi_{\kappa}^{\gamma}}\right)^{2} d \mathbf{k}
$$

which is constant. For a pass-band filter $\widehat{\psi_{\gamma}^{\kappa}}$ defined as a box filter: $11_{[\gamma \kappa]}(\mathbf{k})=\mathbf{k}$ if $\mathbf{k} \in$ $[\gamma, \kappa], 1 \mathrm{l}_{[\gamma \kappa]}(\mathbf{k})=0$ otherwise, this is given by

$$
\frac{d t C_{\zeta}}{(2 \pi)^{d}} \frac{d-1}{d} \int_{\gamma}^{\kappa}\left(\int_{k_{r}=\|\mathbf{k}\|}|\mathbf{k}|^{-\zeta-d} d \mathbf{k}\right) d k_{r} \delta_{i j}=\frac{d t C_{\zeta}}{(2 \pi)^{d}} \frac{d-1}{d} \frac{2 \pi^{d / 2}}{\Gamma\left(\frac{d}{2}\right)} \zeta^{-1}\left(L-\ell_{D}\right) \delta_{i j} .
$$

The constant $C_{\zeta}$ has a dimension of $\ell^{1-\xi / 2} / t$ which follows from dimensional analysis (the energy transfer rate $\epsilon=\mathbb{E}\left(u^{2} / t\right) \sim \ell^{2} / t^{3}$ and $\left.\epsilon \sim C_{\zeta}^{2} \ell^{\xi} / t\right)$. The value of the exponent can be fixed either from theoretical considerations or from experimental values.

\subsubsection{Measurement noise}

As for the observation noise involved in relation (4.2), it is defined from the final uncertainty supplied by the local motion estimator of Section 2 (Eq. (2.13)). The random field is specified as $\gamma_{k}(\underline{\mathbf{x}})=\sigma_{k}(\underline{\mathbf{x}}) \eta_{k}(\underline{\mathbf{x}})$, where $\eta_{k}$ is a Gaussian random field with an exponential covariance

$$
g_{\lambda}\left(\underline{\mathbf{x}}-\underline{\mathbf{x}}^{\prime}\right)=\frac{1}{2 \pi \lambda^{2}} \exp \frac{-\left(\underline{\mathbf{x}}-\underline{\mathbf{x}}^{\prime}\right)^{2}}{2 \lambda^{2}}
$$

and $\sigma_{k}(\underline{\mathbf{x}})$ is defined by the uncertainties associated to the motion measurements at each point $\underline{\mathbf{x}}$. To mitigate the effect of outliers, a Gaussian smoothing with a low variance value is considered. This smoothing avoids the apparition of sharp discontinuities on the uncertainty map. The additive uncertainty $\gamma_{k}$ is hence a Gaussian random field with nonstationary variance $\mathbf{R}(\underline{\mathbf{x}}, \underline{\mathbf{x}})=\sigma_{k}^{2}(\underline{\mathbf{x}})$ and covariance $\mathbf{R}\left(\underline{\mathbf{x}}, \underline{\mathbf{x}}^{\prime}\right)=\sigma_{k}(\underline{\mathbf{x}}) \sigma_{k}\left(\underline{\mathbf{x}}^{\prime}\right) g_{\lambda}\left(\underline{\mathbf{x}}-\underline{\mathbf{x}}^{\prime}\right)$.

Let us now turn to the description of the WEnKF implementation corresponding to our application.

\subsubsection{WEnKF implementation}

The knowledge of the dynamics and observation models together with the corresponding uncertainties we have previously set up constitute all the ingredients needed for the implementation of the WEnKF. The following steps summarize the successive stages of the method:

- At $k=0$, the ensemble of states $\left\{\xi_{0}^{a,(i)}, i=1, \cdots, N\right\}$ are initialized with noisy versions of the SLK vorticity map obtained from the two first images of the sequence using the technique of Section 2. 
- At the current time $k \neq 0$, the ensemble members $\xi_{k-1}^{a,(i)}$ obtained at the previous measurement instant are propagated through the stochastic state dynamics (4.1) to generate the forecast ensemble members $\xi_{k}^{f,(i)}$.

- The EnKF update is then performed with the new observation in order to sample the proposal distribution. The importance sampling weighting (relation (3.11)) based on the likelihood and a resampling process of the particles with respect to those weights are performed. The empirical mean of the analysis ensemble provides the vorticity estimate at time $k$.

- The corresponding velocity field is finally obtained from the Biot-Savart law.

Although this direct WEnKF filtering of the SLK vorticity maps does provide good results as we shall see it, the estimation may fail for long range velocities. To overcome this limitation and to further improve the performance of the WEnKF, we propose in the next section a multiscale extension of WEnKF.

\subsection{Multiscale SLK-WEnKF filtering}

The idea of multiscale WEnKF consists to provide an improved proposal distribution from velocity measurements at different scales. The update step operates iteratively in an incremental coarse-to-fine way by introducing motion measurements obtained at different scales through the Gaussian smoothing parameter $\lambda_{\ell}$ in (2.15). More precisely, at a given scale $\ell \in\left[\ell_{0}, L\right]$ the proposal ensemble is build for each ensemble members from successive analysis steps as follow (in the following for sake of notational convenience we drop the member superscript $(i)$, nevertheless apart from mean vectors all the vector are meant to depend on a given member):

$$
\begin{aligned}
& \xi_{k}^{a, \ell}=\xi_{k}^{f, \ell}+\mathbf{K}_{k}^{\ell}\left(\widetilde{\xi}_{k}^{\ell}+\gamma_{k}^{\ell}-\mathbf{H} \xi_{k}^{f, \ell}\right), \\
& \xi_{k}^{f, \ell}=\xi_{k}^{f, \ell+1}-\bar{\xi}_{k}^{a, \ell+1} .
\end{aligned}
$$

Here, $\xi_{k}^{a, \ell}$ (respectively $\xi_{k}^{f, \ell}$ ) corresponds to the analysis step (respectively forecast) at scale $\ell$ of the particles set and the measurements $\widetilde{\xi}_{k}^{\ell}$ are supplied by the stochastic Lucas and Kanade motion estimates between the backwarped image

$$
\tilde{\mathbf{f}}_{k}^{\ell}\left(\mathbf{X}_{k-1}\right)=\mathbf{f}\left(\mathbf{X}_{k-1}+\sum_{j=\ell}^{L} \mathbf{v}\left(\bar{\xi}_{k}^{a, j+1}\right), k\right),
$$

and image $\mathbf{f}\left(\mathbf{X}_{k-1}, k-1\right)$. The SLK estimator is run for Gaussian windowing standard deviation within the range $\left[\lambda^{\ell_{0}}, \lambda^{L}\right]$. As for the values of parameter $\lambda$, a standard pyramidal multi-scale scheme would correspond to $\lambda^{\ell-1}=0.5 \lambda^{\ell}$. In practice we have founded, as in [32], that smaller values were more efficient and we use $\lambda^{\ell-1}=0.3 \lambda^{\ell}$.

The quantity $\bar{\xi}_{k}^{a, \ell}$ denotes the empirical mean of the analysis ensemble. The initial analysis ensemble is fixed to a null value $\left(\bar{\xi}_{k}^{a, L+1}=0\right)$ and the initial forecast is set to the forecast ensemble computed from the dynamics $\left(\mathrm{x}_{k}^{f,(i), L+1}=\xi_{k}^{f,(i)}\right)$. At the coarsest scale, $L$, 
the motion estimation is hence carried out on the original images $\mathbf{f}_{k-1}$ and $\mathbf{f}_{k}$. These estimates are computed at a rough scale through a large value of the gaussian window function $g_{\lambda^{L}}$ (2.14). At finer scales the motion estimation is performed for a decreasing value of this parameter on a pair of images constituted by the initial image $\mathbf{f}_{k-1}$ and the image $\tilde{\mathbf{f}}_{k}^{\ell}$ obtained from backward registration of image $\mathbf{f}_{k}$ with respect to the analyzed motion field at the previous scale, $\mathbf{v}\left(\bar{\xi}_{k}^{a, \ell+1}\right)$.

At each scale, the Gaussian random fields, $\gamma^{\ell}$, attached to the measurements perturbations involved in the analysis step (4.6a) are drawn with the uncertainties provided by the stochastic Lucas and Kanade formulation computed from the couple of images $\left(\mathbf{f}_{k-1}, \widetilde{\mathbf{f}}_{k}^{\ell}\right)$ and the current analysis ensemble (2.13). Let us note that compared to the previous single scale filtering where the proposal was based on a single ensemble Kalman update, here several updates associated to different Kalman gains computed from observations at different scales are considered. In the experimental section, three successive scales will be considered in such a filtering. The final proposal corresponds to the sum of the analysis ensemble obtained at the different scales: $\xi_{k}^{a}=\sum_{\ell=\ell_{0}}^{L} \xi_{k}^{a, \ell}$. In the same way as the previous filter, these ensemble members are then resampled according to the importance weights computed from the likelihood associated to the original couple of images $\left(\mathbf{f}_{k-1}, \mathbf{f}_{k}\right)$.

The multiscale version of the Weighted ensemble Kalman filter (WEnKF) procedure can be summarized by the Algorithm 4.1.

Algorithm 4.1: Multiscale WEnKF algorithm, one iteration

Require: Ensemble at instant $k-1:\left\{\xi_{k-1}^{(i)}, i=1, \cdots, N\right\}$

Ensure: Ensemble at time $k:\left\{\xi_{k}^{(i)}, i=1, \cdots, N\right\}$

\section{EnKF step:}

Compute the forecast ensemble $\xi_{k}^{f}$ according to (3.8);

$\bar{\xi}_{k}^{a, L+1}=0 ; \xi_{k}^{f, L+1}=\xi_{k}^{f} ; \bar{\xi}_{k}^{a}=0 ;$

for $\ell=L \rightarrow \ell_{0}$ do

Compute the backward registered image $\tilde{\mathbf{f}}_{k}^{\ell}\left(\mathbf{X}_{k-1}\right)=\mathbf{f}\left(\mathbf{X}_{k-1}+\mathbf{v}\left(\bar{\xi}_{k}^{a}\right), k\right)$;

Compute the SLK estimate between images $\mathbf{f}_{k-1}$ and $\widetilde{\mathbf{f}}_{k}^{\text {; }}$;

Get the observation covariance $\mathbf{R}^{\ell}$;

Draw $N$ samples of Gaussian random fields $\gamma_{k}^{\ell} \sim \mathscr{N}\left(0, \mathbf{R}^{\ell}\right)$;

Compute the analysis ensemble at scale $\ell$ and the forecast residual from

$\xi_{k}^{f, \ell}=\xi_{k}^{f, \ell+1}-\bar{\xi}_{k}^{a, \ell+1}$

$\xi_{k}^{a, \ell}=\xi_{k}^{a, \ell+1}+\xi_{k}^{f,(i), \ell}+\mathbf{K}_{k}^{\ell}\left(\widetilde{\xi}_{k}^{\ell}+\boldsymbol{r}_{k}^{\ell}-\mathbf{H} \xi_{k}^{f, \ell}\right)$;

$\bar{\xi}_{k}^{a, \ell}=\frac{1}{N} \sum_{i=1}^{N} \xi_{k}^{a, \ell}$;

$\bar{\xi}_{k}^{a}=\bar{\xi}_{k}^{a, \ell}$

end for

Compute the weights $w_{k}^{(i)}$ according to (3.11);

Resample: For $j=1, \cdots, N$ sample with replacement index $I(j)$ from discrete probability $\left\{w_{k}^{(i)}, i=1, \cdots, N\right\}$ over $\{1, \cdots, N\}$ and set $\xi_{k}^{(j)}=\xi_{k}^{a, I(j)}$. 


\section{Experimental results and comparisons}

In this section, we first validate and compare our technique with some state-of-theart approaches on a synthetic sequence built from a Direct Numerical Simulation (DNS) corresponding to a $2 \mathrm{D}$ sustained turbulence. In a second step, we test our approach on a real fluid sequence.

\subsection{Synthetic DNS sequence}

We present the results obtained by the application of the single scale and the multiscale WEnKF denoted as 1L-WEnKF and 3L-WEnKF respectively as the latter has been applied on a set of 3 three successive scale ranges. Those filters have been compared with stateof-the-art fluid motion estimators $[14,26,33]$ on a sequence of 100 images depicting a 2D turbulent flow seeded with particles. The flow has been computed with a direct numerical simulation of the Navier-Stokes equations for a Reynolds number of 3000. This sequence can be downloaded at http://www. fluid.irisa.fr.

Quantitative comparisons of the different estimation techniques with the ground truth in terms of the Root-Mean-Square-Error (RMSE) of vorticity and velocity are both presented in Fig. 3.

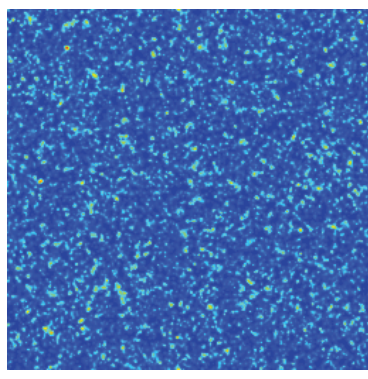

(a)

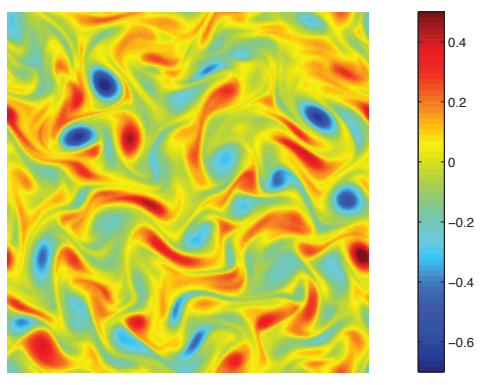

(b)

Figure 2: (a): Particle image (50th of the sequence) and (b): the associated true vorticity map.

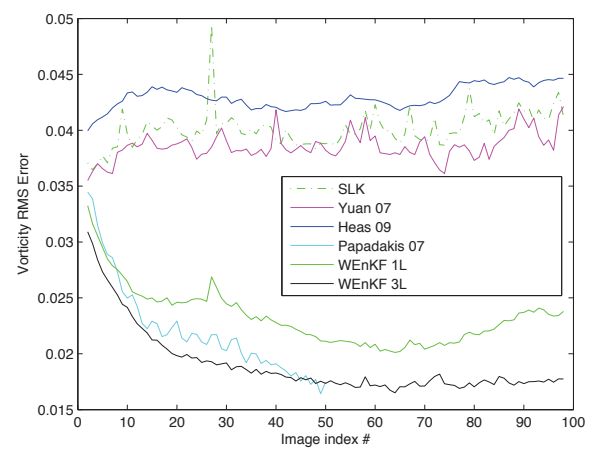

(a)

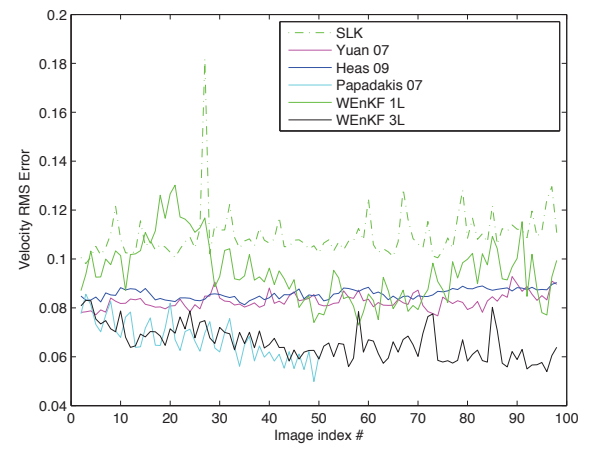

(b)

Figure 3: Comparison with State of the Art: RMSE in (a) Vorticity; (b) Motion field. 
For such particle images, it is noticeable to observe from Fig. 3(a), that the RMSE in vorticity of the SLK approach is close to state of art approaches [14,33], though the RMSE values in velocity are higher (Fig. 3(b)). The RMSE in vorticity by assimilating the SLK observation through $1 \mathrm{~L}-\mathrm{WEnKF}$ is much lower, while the error in terms of velocity estimates is close to the approach of Yuan et al. [33]. However, the 3L-WEnKF assimilation shows better results both in terms of vorticity or velocity. These errors are lower than all the fluid motion estimators that have been tested and are at the same level as the errors provided by the batch variational assimilation techniques $[26]$ (which corresponds thus to a smoothing filter as opposed to a recursive filter as in our case). Let us note that the RMSE criterion corresponds only to a large scale indicator as the errors on small scales motion structures contribute to a small amount to the whole error energy. Besides, the vorticity map is very sensitive to the presence of noise in the velocity fields. The higher errors in vorticity of the motion estimators may be observed from the different vorticity maps plotted in Fig. 4. Only data assimilation techniques (either variational or based on stochastic filtering as in this work) enable to recover vorticity map that are closer to the ground truth maps even in the finer small scales details. The presence of noisy structures in the vorticity maps provided by all the motion measurement techniques reflects the emergence of small scale dynamical inconsistencies along time.

For a visual comparison we show in Fig. 4 the vorticity maps obtained by the different methods for the 50th images of the sequence. We in addition depicted the estimation at 3 various scales issued from SLK in order to observe the evolution of the structures along scales (Fig. 4(g-h-i)). From these images, it appears that the vorticity estimated by the 3L-WEnKF assimilation (Fig. 4(f)) is less noisy and exhibits much finer scales vortical structures. The quantitative gain obtained by the proposed 3L-WEnKF method indicates that such fine structures are physically consistent. This constitutes a key contribution of the paper.

Let us now analyze the behavior of those techniques for real data.

\subsection{Real fluid sequence}

Our next set of results corresponds to a real world image sequence of a 2D turbulence generated from the wake of a soap film behind a comb. The flow is visualized through a Schlieren technique at a rate of 2500 frames per second. This experiment and the image acquisition have been performed at the Fluid dynamics laboratory of the University of Buenos Aires. A typical image of the sequence is shown in Fig. 5 in false color. In this experiment, we have compared a pure SLK estimation with its filtering using $1 \mathrm{~L}$ WEnKF and 3L WEnKF techniques. The estimated vorticity maps and velocity fields corresponding to SLK, 1L WEnKF and 3L WEnKF are shown in Figs. 6(a), (b) and (c), respectively. We note that though the 1L-WEnKF assimilation of SLK brings out some details at a smaller scale than the SLK measurements, the 3L-WEnKF assimilation recovers even finer details, which is a very good behavior. In addition, they are physically consistent since the extracted

\footnotetext{
Due to technical limits associated to batch techniques used in variational data assimilation, the results were unfortunately available only for 50 images.
} 


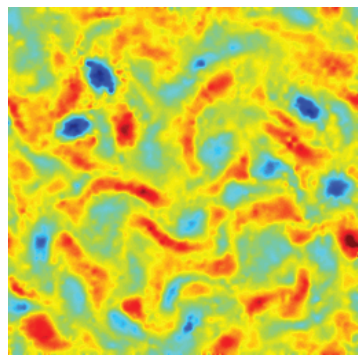

(a)

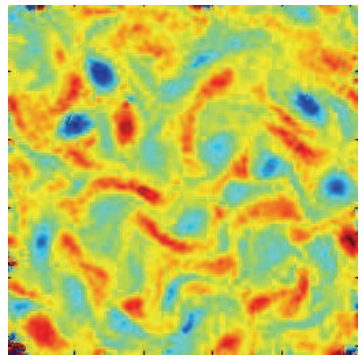

(d)

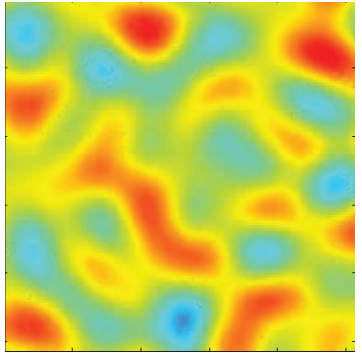

$(\mathrm{g})$

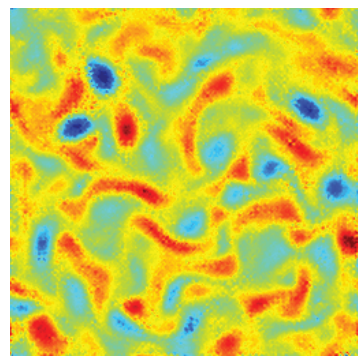

(b)

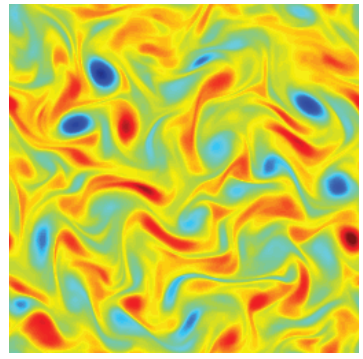

(e)

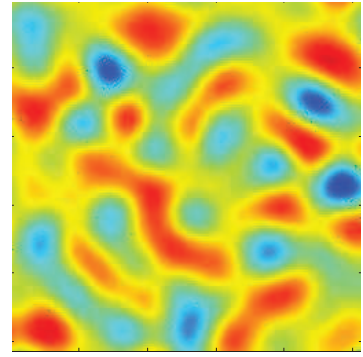

(h)

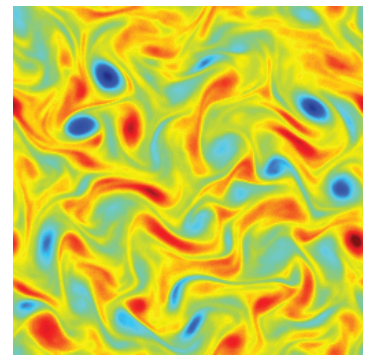

(c)

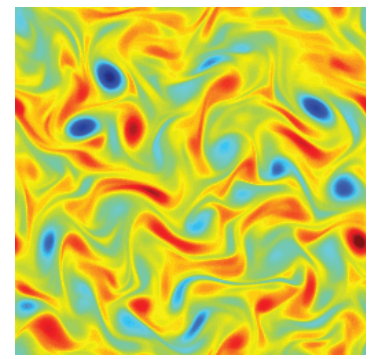

(f)

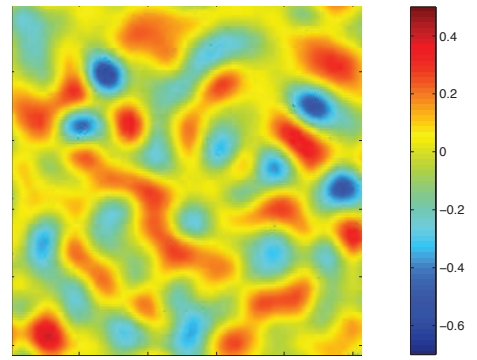

(i)

Figure 4: Estimated vorticity maps for (a) Yuan et al.; (b) Heas et al. [14]; (c) Papadakis et al. [26]; (d) SLK; (e) 1-Level WEnKF assimilation; (f) 3-Levels WEnKF assimilation with the representation color scale. Last line: intermediate scales estimations during the SLK estimation process for three various scales (from coarser scale in $(\mathrm{g})$ to finer in (i)).

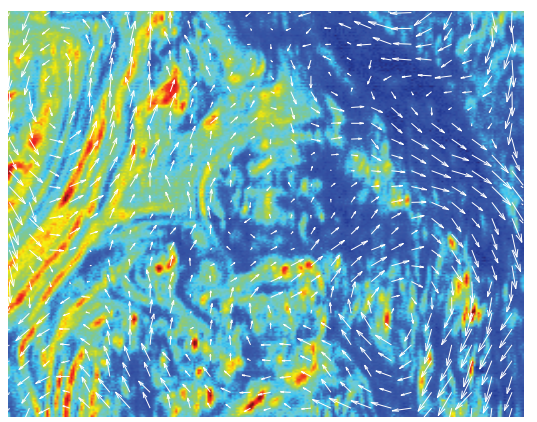

Figure 5: Real image sequence of a 2D turbulent flow of a soap film. 

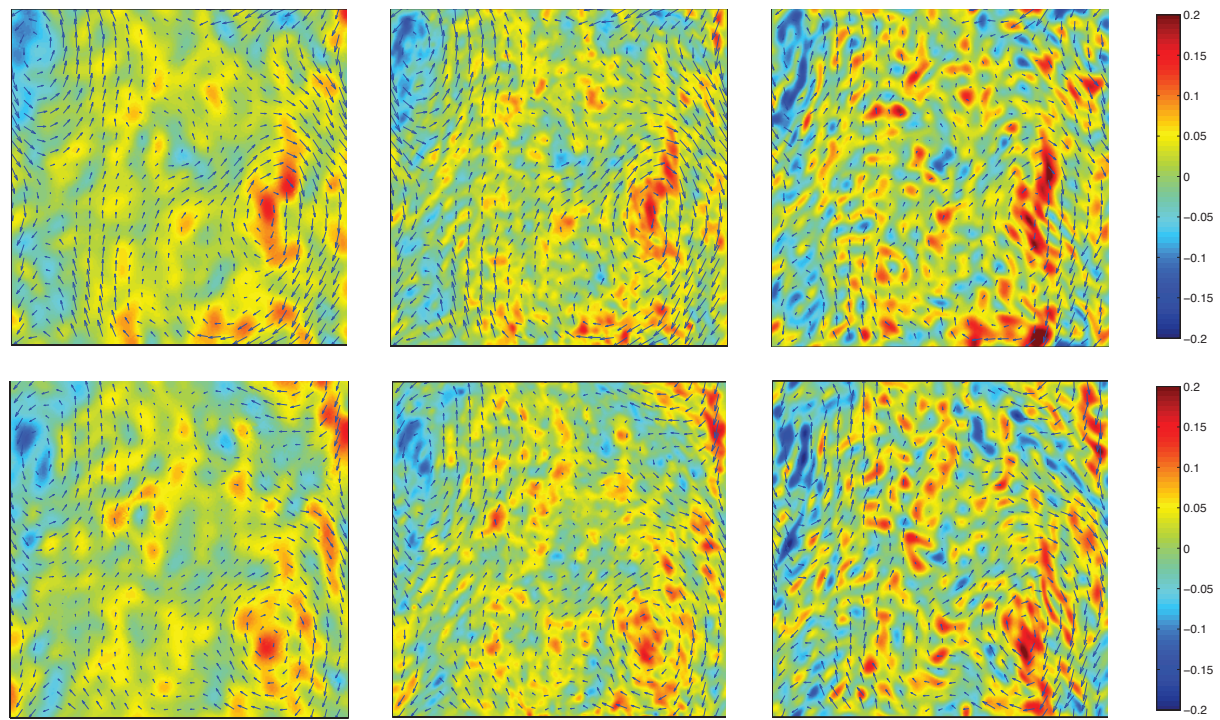

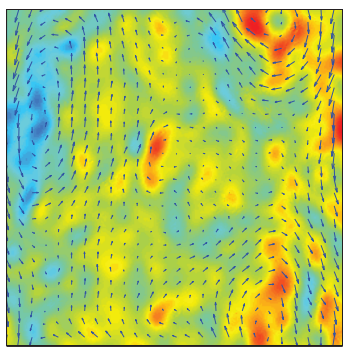

(a)

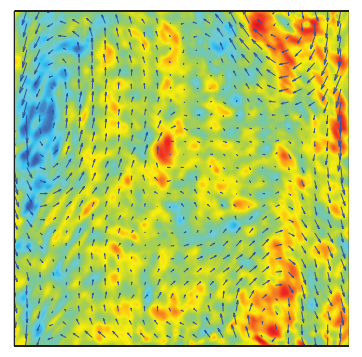

(b)

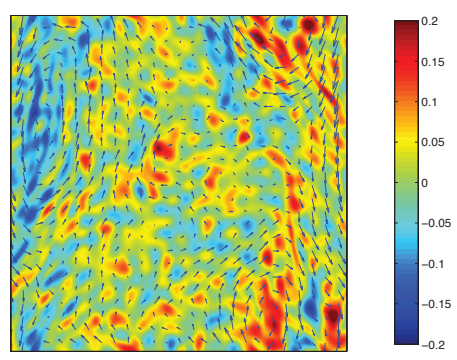

(c)

Figure 6: Vorticity maps with their corresponding velocity fields, (a) SLK; (b) 1L- WEnKF; (c) 3LWEnKF for images 76,86 and 96 (first, second and third row respectively).

patterns are representative of this kind of flows where details such as small scale vorticity filaments and smaller vortices are revealed. Let us also remark that the results observed in terms of velocity (see the motion fields superimposed on the vorticity fields in Fig. 4(gh-i)) remains consistent and are close together when interpreted at a larger scale, which demonstrates the power of the WEnKF methods to extract fine scale structures from larger scales observations and a dynamical model.

\section{Conclusions}

In this paper, we have proposed an efficient multiscale extension of the Weighted Ensemble Kalman filter for fluid flow motion estimation problem. This filter is a particle filter relying on a proposal distribution built from the ensemble Kalman filtering mechanism. The particular instance we considered here incorporates measurements issued from a stochastic extension of the Lucas and Kanade estimator. It benefits from a natural multi- 
scale formulation of the motion estimator. The overall multiscale data assimilation filter is defined in an incremental way.

We have tested our approach on synthetic and real fluid flow sequences. As for the the former situation, we have also compared our method with several state-of-the-art fluid motion estimators. From these experiences it can be pointed out that on the basis of SLK motion estimates and their associated uncertainties, the overall process presented in this article enables to recover finer scale structures and therefore, to dramatically improve the quality of the results. Considering only few levels of this hierarchical filter enables indeed to improve the errors of the corresponding single level filter. This promising behavior makes the algorithm an appealing technique for fluid motion estimation in image sequences. As for the computational load of the technique proposed, it relies on a local incremental motion estimator that is only slightly more expansive than the original Lucas and Kanade estimator due to the uncertainty computation. This estimator could be coded in real time on a Graphics Processing Unit (GPU) [4]. The Weighted ensemble particle filter is intrinsically parallel and could be very efficiently implemented on a grid computer, in order to get small computational time. In this study, all the experiments have been performed through a very inefficient Matlab code. In the future we plan to investigate an efficient implementation of this code together with a 3D Navier Stokes.

Acknowledgments The authors acknowledge the support of the French Agence Nationale de la Recherche (ANR), under grant PREVASSEMBLE (ANR-08-COSI-012).

\section{Appendix A: Lucas-Kanade technique}

Starting from the from the optical-flow constraint equation:

$$
\frac{\partial \mathbf{f}(\underline{\mathbf{x}}, t)}{\partial t}+\mathbf{v}(\underline{\mathbf{x}}, t) \cdot \nabla \mathbf{f}(\underline{\mathbf{x}}, t)=0
$$

the Lucas-Kanade estimator assumes the velocity $\mathbf{v}(\underline{\mathbf{x}}, t)$ at a given position $\underline{\mathbf{x}}$ to be homogeneous inside a gaussian window $g_{\lambda}$ of variance $\lambda$ centered in $\underline{\mathbf{x}}$. Therefore, the estimation of $\mathbf{v}(\underline{\mathbf{x}}, t)$ reads:

$$
\begin{aligned}
\mathbf{v} & =\min _{\mathbf{v}^{\star}=(u, v)^{T}} \int_{\Omega} \mathscr{J}\left(\mathbf{v}^{\star}\right) d \underline{\mathbf{x}} \\
& =\min _{\mathbf{v}^{\star}=(u, v)^{T}} \int_{\Omega}\left[g_{\lambda}(\cdot) *\left(\frac{\partial \mathbf{f}(\cdot, t)}{\partial t}+\mathbf{v}^{\star}(\underline{\mathbf{x}}, t) \cdot \nabla \mathbf{f}(\cdot, t)\right)^{2}\right](\underline{\mathbf{x}}) d \underline{\mathbf{x}} .
\end{aligned}
$$

The Euler-Lagrange equation associated to the cost-function $\mathscr{J}\left(\mathbf{v}^{\star}\right)$ reads (omitting indexes for clarity reasons):

$$
2 g_{\lambda} * \nabla \mathbf{f}\left(\nabla \mathbf{f}^{T} \mathbf{v}+\mathbf{f}_{t}\right)=0
$$

where $\nabla \mathbf{f}=\left[\mathbf{f}_{x}, \mathbf{f}_{y}\right]^{T}=[\partial \mathbf{f} / \partial x, \partial \mathbf{f} / \partial y]^{T}$ and $\mathbf{f}_{t}=\partial \mathbf{f} / \partial t$. The value of $\mathbf{v}(\underline{\mathbf{x}}, t)$ being constant inside the convolution window $g_{\lambda}$, one gets:

$$
2\left(g_{\lambda} * \nabla \mathbf{f} \nabla \mathbf{f}^{T}\right) \mathbf{v}+2 g_{\lambda} * \nabla \mathbf{f}\left(\mathbf{f}_{t}\right)=0
$$


and finally

$$
\mathbf{v}=-\left(g_{\lambda} *\left[\begin{array}{cc}
\mathbf{f}_{x}^{2} & \mathbf{f}_{x} \mathbf{f}_{y} \\
\mathbf{f}_{x} \mathbf{f}_{y} & \mathbf{f}_{y}^{2}
\end{array}\right]\right)^{-1} g_{\lambda} *\left[\begin{array}{c}
\mathbf{f}_{x} \mathbf{f}_{t} \\
\mathbf{f}_{y} \mathbf{f}_{t}
\end{array}\right]
$$

\section{Appendix B: Expectation of a function of a stochastic process}

The conditional expectation given $\mathbf{X}_{t-1}$ of any function $\Psi\left(\mathbf{X}_{t}, t\right)$ of a stochastic process defined through Itô diffusion (2.5) and discretized through an Euler scheme $\mathbf{X}_{t}=\mathbf{X}_{t-1}+$ $\mathbf{v}\left(\mathbf{X}_{t-1}\right) d t+\boldsymbol{\Sigma}^{1 / 2}\left(B_{t+1}-B_{t}\right)$ may be written as:

$$
E\left(\Psi\left(\mathbf{X}_{t}, t\right) \mid \mathbf{X}_{t-1}\right)=\int_{\mathbb{R}} \Psi\left(\mathbf{X}_{t}, t\right) p\left(\mathbf{X}_{t} \mid \mathbf{X}_{t-1}\right) d \mathbf{X}_{t},
$$

where $\boldsymbol{\Sigma}$ is a fixed matrix-valued function. As the process $\mathbf{X}_{t}$ is known up to the Brownian motion $\Sigma^{1 / 2} d \mathbf{B}_{t}$, the probability $p\left(\mathbf{X}_{t} \mid \mathbf{X}_{t-1}\right)$ is a multidimensional Gaussian of variance $\Sigma \delta t(\delta t=1$ here $)$ and we get:

$$
\begin{aligned}
& E\left(\Psi\left(\mathbf{X}_{t}\right) \mid \mathbf{X}_{t-1}\right) \\
= & \frac{1}{\sqrt{2 \pi} \operatorname{det}(\Sigma)^{\frac{1}{2}}} \int_{\mathbb{R}} \Psi\left(\mathbf{X}_{t}, t\right) \exp \left(-\left(\mathbf{X}_{t-1}+\mathbf{v}-\mathbf{X}_{t}\right) \Sigma^{-1}\left(\mathbf{X}_{t-1}+\mathbf{v}-\mathbf{X}_{t}\right)\right) d \mathbf{X}_{t} .
\end{aligned}
$$

By a change of variable $\mathbf{Y}_{t}=\mathbf{X}_{t-1}+\mathbf{v}-\mathbf{X}_{t}$, this expectation can be written as:

$$
\begin{aligned}
E\left(\Psi\left(\mathbf{X}_{t}, t\right) \mid \mathbf{X}_{t-1}\right) & =\frac{1}{\sqrt{2 \pi} \operatorname{det}(\boldsymbol{\Sigma})^{1 / 2}} \int_{\mathbb{R}} \Psi\left(\mathbf{X}_{t-1}+\mathbf{v}-\mathbf{Y}_{t}, t\right) \exp \left(-\mathbf{Y}_{t} \boldsymbol{\Sigma}^{-1} \mathbf{Y}_{t}\right) d \mathbf{Y}_{t} \\
& =\left(\widetilde{\Psi}(\cdot, t) * g_{\Sigma}(\cdot)\right)\left(\mathbf{X}_{t}\right),
\end{aligned}
$$

where $g_{\Sigma}\left(\mathbf{X}_{t}\right) \sim \mathscr{N}\left(\mathbf{X}_{t}, \boldsymbol{\Sigma}^{1 / 2}\right)$ is a multidimensional Gaussian of mean $\mathbf{X}_{t}$ with covariance $\Sigma$ and $\widetilde{\Psi}\left(\mathbf{X}_{t}, t\right)=\Psi\left(\mathbf{X}_{t-1}+\mathbf{v}, t\right)$.

\section{References}

[1] S. Baker, D. Scharstein, J. P. Lewis, S. Roth, M. J. Black And R. Szeliski, A database and evaluation methodology for optical flow, Int. J. Comput. Vision, 92(1) (2010), pp. 1-31.

[2] J. Barron, D. Fleet and S. Beauchemin, Performance of optical flow techniques, Int. J. Comput. Vision, 12(1) (1994), pp. 43-77.

[3] A. Becciu, R. Duits, B. Janssen, L. Florack and H. van Assen, Cardiac motion estimation using covariant derivatives and Helmholtz decomposition, In MICCAI workshop LNCS 7085, pages 263-273, Heidelberg, 2012.

[4] F. Champagnat, A. Plyer, G. Le Besnerais, B. Leclaire and Y. Le Sant, How to calculate dense piv vector fields at video rate, In 8th Int. Symp. On Particle Image Velocimetry-PIV09, Melbourne, Australia, 2009.

[5] T. Corpetti, P. Heas, E. Memin and N. Papadakis, Pressure image asimilation for atmospheric motion estimation, Tellus, 61A (2009), pp. 160-178. 
[6] T. Corpetti, D. Heitz, G. Arroyo, E. Memin and A. Santa-Cruz, Fluid experimental flow estimation based on an optical-flow scheme, Experiments Fluids, 40 (2006), pp. 80-97.

[7] R. Duits, B. Janssen, A. Becciu And H. van Assen, A variational approach to cardiac motion estimation based on covariant derivatives and multi-scale helmholtz decomposition, Quart. Appl. Math., American Mathematical Society, to appear, 2012.

[8] F. Elliot, D. Horntrop And A. Majda, A Fourier-wavelet Monte Carlo method for fractal random fields, J. Comput. Phys., 132(2) 91997), pp. 384-408.

[9] G. Evensen, Sequential data assimilation with a non linear quasi-geostrophic model using Monte Carlo methods to forecast error statistics, J. Geophys. Res., 99(C5)(10) (1994), pp. 143-162.

[10] G. EvENSEN, The ensemble Kalman filter, theoretical formulation and practical implementation, Ocean Dyn., 53(4) (2003), pp. 343-367.

[11] L. Florack, W. Niessen and M. Nielsen, The intrinsic structure of optic flow incorporating measurement duality, Int. J. Comput. Vision, 27(3) (1998), pp. 263-286.

[12] B. Galvin, B. McCane, K. Novins, D. Mason and S. Mills, Recovering motion fields: an analysis of eight optical flow algorithms, In Proc. British Mach. Vis. Conf., Southampton, 1998.

[13] N. J. Gordon, D. J. SAlmond And A. F. M. Smith, Novel approach to non-linear/non-Gaussian Bayesian state estimation, IEEE Proc. F, 140(2) (1993).

[14] P. Heas, E. Memin, D. Heitz and P. Mininni, Bayesian selection of scaling laws for motion modeling in images, In Proc. Int. Conf. Computer Vision, 2009.

[15] P. Heas, E. Memin, D. Heitz and P. Mininni, Power laws and inverse motion modeling: application to turbulence measurements from satellite images, Tellus-A, 64 (2012), pp. 1-24.

[16] D. Heitz, E. Memin and C. Schnoerr, Variational fluid flow measurements from image sequences: synopsis and perspectives, Exp. Fluids, 48(3) (2010), pp. 369-393.

[17] B. Horn And B. Schunck, Determining optical flow, Artif. Intell., 17 (1981), pp. 181-203.

[18] R. E. Kalman, A new approach to linear filtering and prediction problems, Trans. ASME J. Basic Eng., 82 (1960), pp. 35-45.

[19] R. Kraichnan, Small-scale structure of a scalar field convected by turbulence, Phys. Fluids, pages 945-963, 1968.

[20] F. Le Gland, V. Monbet And V. D. Tran, Large sample asymptotics for the ensemble Kalman filter, In D. Crisan and B. Rozovskii, editors, Handbook on Nonlinear Filtering, Oxford University Press, 2011.

[21] T. LindEBERG, Scale-space theory: a basic tool for analysing structures at different scales, J. Appl. Stat., 21(2) (1994), pp. 224-270.

[22] B. LuCAS AND T. KANADE, An iterative image registration technique with an application to stereovision, In Int. Joint Conf. Artificial Intel (IJCAI), pages 674-679, 1981.

[23] A. Majda AND P. KRAmer, Simplified models for turbulent diffusion: theory, numerical modelling and physical phenomena, Phys. Report, 314 (1999), pp. 237-574.

[24] T. NiR, A BRUCKSTEIn AND R. Kimmel, Over-parameterized variational optical flow, Int. J. Comput. Vision, 76(2) 92008), pp. 205-216.

[25] B. OKSEndal, Stochastic Differential Equations, Spinger-Verlag, 1998.

[26] N. Papadakis and E. Memin, An optimal control technique for fluid motion estimation, SIAM J. Imag. Sci., 1(4) (2008), pp. 343-363.

[27] N. Papadakis, E. Memin, A. Cuzol and N. Gengembre, Data assimilation with the weighted ensemble Kalman filter, Tellus-A, 62(5) (2010), pp. 673-697.

[28] N. Papenberg, A. Bruhn, T. Brox, S. Didas and J. Weickert, Highly accurate optic flow computation with theoretically justified warping, IJCV, 67(2) (2006), pp. 141-158.

[29] P. Ruhnau, T. Kohlberger, C. Schnoerr and H. Nobach, Variational optical flow estimation for particle image velocimetry, Exp. Fluids, 38 (2005), pp. 21-32. 
[30] C. Snyder, T. Bengtsson, P. Bickel And J. Anderson, Obstacles to high-dimensional particle filtering, Monthly Weather Rev., 136(12) (2008), pp. 4629-4640.

[31] D. Sun, E. Sudderth AND M. J. Black, Layered segmentation and optical flow estimation over time, In Proc. Conf. Comput. Vision Pattern Rec., Providence, Rhode Island, 2012.

[32] Y. WU, T. KANADE, C. LI AND J. CoHN, Image registration using wavelet-based motion model, Int. J. Comput. Vision, 38(2) (2000), pp. 129-152.

[33] J. Yuan, C. Schnoerr and E. Memin, Discrete orthogonal decomposition and variational fluid flow estimation, J. Math. Imag. Vision, 28(1) (2007), pp. 67-80. 